\title{
Urethra Part
}

National Cancer Institute

\section{Source}

National Cancer Institute. Urethra Part. NCI Thesaurus. Code C160608.

A named segment of the urethra. 